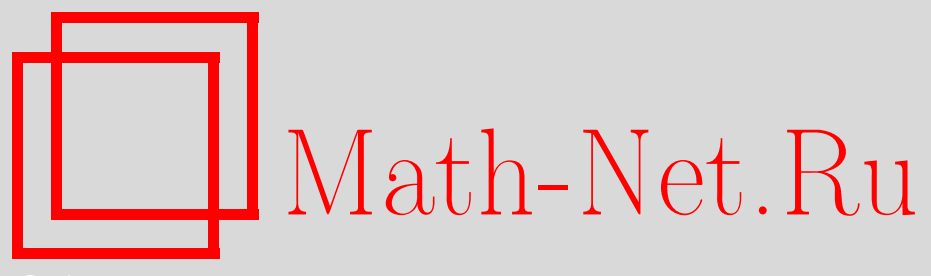

X. Фернандес Нуньес, В. Гарсия Фуертес, А. М. Переломов, Явные вычисления низколежащих собственных функций для квантовой тригонометрической модели Калоджеро-Сазерленда, связанной с исключительной алгеброй $E_{7}, T M \Phi, 2008$, том 154 , номер 2, 283-293

DOI: https://doi.org/10.4213/tmf6169

Использование Общероссийского математического портала Math-Net.Ru подразумевает, что вы прочитали и согласны с пользовательским соглашением http://www . mathnet.ru/rus/agreement

Параметры загрузки:

IP : 35.173 .137 .237

26 апреля 2023 г., 14:24:36

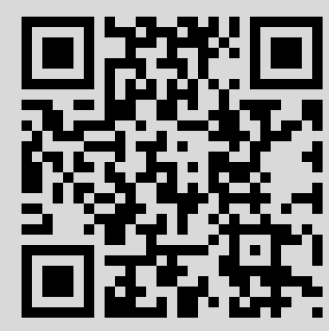


(C) 2008 г. $\quad$ Х. Фернандес Нуньес*, В. Гарсиа Фуертес*, А. М. Переломов ${ }^{*} \dagger$

\section{ЯВНЫЕ ВЫЧИСЛЕНИЯ НИЗКОЛЕЖАЩИХ СОБСТВЕННЫХ ФУНКЦИЙ ДЛЯ КВАНТОВОЙ ТРИГОНОМЕТРИЧЕСКОЙ МОДЕЛИ КАЛОДЖЕРО-САЗЕРЛЕНДА, СВЯЗАННОЙ С ИСКЛЮЧИТЕЛЬНОЙ АЛГЕБРОЙ $E_{7}$}

В предыдущей работе авторов были изучены характеры и ряды Клебша-Гордана для исключительной алгебры Ли $E_{7}$ на основе их связи с квантовым тригонометрическим гамильтонианом Калоджеро-Сазерленда с константой $\kappa=1$. В данной работе мы обобщаем этот подход на случай произвольной константы связи.

Ключевые слова: интегрируемые системы, модели Калоджеро-Сазерленда, исключительные алгебры Ли, теория представлений, ортогональные полиномы.

\section{1. ВВЕДЕНИЕ}

Модели Калоджеро-Сазерленда [1], [2], связанные с системами корней простых алгебр Ли [3]-[5], глубоко исследовались в течение последних двух десятилетий. Изначально введенные на основе чисто теоретических соображений, модели этого класса нашли тем не менее целый ряд актуальных применений в таких разных областях, как физика конденсированного состояния, суперсимметричная теория Янга-Миллса и физика черных дыр. С математической точки зрения интересная особенность квантового варианта моделей этого типа состоит в том, что их собственные энергетические функции дают естественное обобщение нескольких типов гипергеометрических функций на случай многих переменных. Для потенциала $v(q)=\kappa(\kappa-1) \sin ^{-2} q$ и при специальных значениях константы связи $\kappa$ эти собственные функции связаны с некоторыми ортогональными функциональными системами, представляющими особый интерес в теории алгебр Ли и симметрических пространств: так, при $\kappa=1$ получаются характеры неприводимых представлений

*Departamento de Física, Facultad de Ciencias, Universidad de Oviedo, Oviedo, Spain

${ }^{\dagger}$ Институт теоретической и экспериментальной физики, Москва, Россия. E-mail: perelomo@dftuz.unizar.es 
алгебры, а при $\kappa=0$ возникают соответствующие мономиальные симметрические функции. Другие значения $\kappa$ приводят к зональным сферическим функциям в симметрических пространствах, связанных с данной алгеброй Ли. В частности, для $E_{7}$ значение $\kappa=1 / 2$ дает такие функции для симметрического пространства $\mathrm{EV}^{*}$ [5], [6]. Гамильтониан Калоджеро-Сазерленда появляется при этом как естественное унифицирующее средство для вычисления всех этих объектов.

Гамильтониан Калоджеро-Сазерленда, связанный с системой корней простой алгебры Ли, можно записать как дифференциальный оператор второго порядка, переменные которого являются характерами фундаментальных представлений алгебры. Как было показано в работах [7]-[9] и позднее в [10]-[16], такой подход позволяет разработать определенные систематические процедуры для решения уравнения Шредингера и определить такие важные свойства собственных функций, как рекурсионные соотношения или производящие функции для некоторых их подмножеств. Данный подход был применен к классическим алгебрам типа $A_{n}$ и $D_{n}$, к исключительной алгебре $E_{6}$, а недавно и к $E_{7}$ при специальном значении константы связи, когда собственные функции пропорциональны характерам неприводимых представлений алгебры. Цель настоящей работы - показать, как обобщить подход работы [17] на произвольные значения константы связи и распространить некоторые из частных результатов, полученных в [17], на общий случай.

\section{2. ГАМИЛЬТОНИАН КАЛОДЖЕРО-САЗЕРЛЕНДА ДЛЯ $E_{7}$ В ВЕЙЛЬ-ИНВАРИАНТНЫХ ПЕРЕМЕННЫХ}

Тригонометрическая модель Калоджеро-Сазерленда, связанная с системой корней $\mathcal{R}$ алгебры Ли ранга $r$ с однократными связями между корнями, является квантовой системой в евклидовом пространстве $\mathbb{R}^{r}$, задаваемой стандартным оператором Гамильтона

$$
H=\frac{1}{2} \sum_{j=1}^{r} p_{j}^{2}+\kappa(\kappa-1) \sum_{\alpha \in \mathcal{R}^{+}} \sin ^{-2}(\alpha, q),
$$

где $q=\left(q_{j}\right)$ - декартова система координат, $p_{j}=-i \partial_{q_{j}}, \mathcal{R}^{+}-$множество положительных корней алгебры, $(\cdot, \cdot)$ - скалярное произведение в $\mathbb{R}^{r}$, а $\kappa$ - константа связи. Ненормированная волновая функция основного состояния имеет вид

$$
\Psi_{0}^{\kappa}(q)=\prod_{\alpha \in \mathcal{R}^{+}} \sin ^{\kappa}(\alpha, q)
$$

а возбужденные состояния индексируются старшими весами $\mu=\sum m_{i} \lambda_{i} \in P^{+}$ (здесь $P^{+}-$конус доминантных весов) неприводимых представлений алгебры, т.е. набором $\mathbf{m}=\left(m_{1}, \ldots, m_{r}\right)$ из $r$ неотрицательных целых чисел. При поиске решений $\Psi_{\mathbf{m}}^{\kappa}$ уравнения Шредингера в виде

$$
\Psi_{\mathbf{m}}^{\kappa}(q)=\Psi_{0}^{\kappa}(q) \Phi_{\mathbf{m}}^{\kappa}(q)
$$

мы приходим к задаче на собственные значения

$$
\Delta^{\kappa} \Phi_{\mathbf{m}}^{\kappa}=\varepsilon_{\mathbf{m}}(\kappa) \Phi_{\mathbf{m}}^{\kappa},
$$


где $\Delta^{\kappa}-$ линейный дифференциальный оператор,

$$
\Delta^{\kappa}=-\frac{1}{2} \sum_{j=1}^{r} \partial_{q_{j}}^{2}-\kappa \sum_{\alpha \in \mathcal{R}^{+}} \operatorname{ctg}(\alpha, q)\left(\alpha, \nabla_{q}\right),
$$

а собственные значения имеют вид

$$
\varepsilon_{\mathbf{m}}(\kappa)=2(\mu, \mu+2 \kappa \rho)
$$

где $\rho$ - вектор Вейля, $\rho=\sum_{\alpha \in \mathcal{R}^{+}} \alpha / 2$.

В силу вейлевской симметрии гамильтониана при решении задачи (4) на собственные значения удобно выразить оператор $\Delta^{\kappa}$ в терминах набора независимых вейль-инвариантных (далее $W$-инвариантных) переменных $z_{k}=\chi_{l_{k}}(q)$ - характеров неприводимых представлений алгебры. Оператор $\Delta^{\kappa}$ в терминах переменных $z$ имеет вид

$$
\Delta^{\kappa}=\sum_{j, k} a_{j k}(z) \partial_{z_{j}} \partial_{z_{k}}+\sum_{j}\left[b_{j}^{0}(z)+\kappa b_{j}^{1}(z)\right] \partial_{z_{j}},
$$

однако нахождение его коэффициентов путем непосредственной замены переменных исключительно громоздко. Как объяснялось в [17], существует иная процедура, основанная на вычислении квадратичного ряда Клебша-Гордана и характеров второго порядка алгебры $E_{7}$. В указанной работе мы применили эту процедуру для вычисления $a_{j k}(z)$ и $b_{j}^{0}(z)+b_{j}^{1}(z)$, тем самым найдя оператор $\Delta^{\kappa}$ в специальном случае $\kappa=1$. Полагая, что это будет полезно читателю, воспроизведем здесь список коэффициентов $a_{j k}(z)$ :

$$
\begin{aligned}
& a_{11}(z)=4\left(-19-10 z_{1}+z_{1}^{2}-z_{3}-5 z_{6}\right), \\
& a_{12}(z)=2\left(-7 z_{2}+2 z_{1} z_{2}-5 z_{5}-19 z_{7}-13 z_{1} z_{7}\right) \text {, } \\
& a_{13}(z)=2\left(10-14 z_{1}-19 z_{1}^{2}+13 z_{3}+3 z_{1} z_{3}-3 z_{4}-4 z_{6}-9 z_{1} z_{6}-6 z_{2} z_{7}+9 z_{7}^{2}\right) \text {, } \\
& a_{14}(z)=2\left(-10-2 z_{1}+18 z_{1}^{2}-7 z_{2}^{2}-24 z_{3}-6 z_{1} z_{3}+14 z_{4}+4 z_{1} z_{4}-4 z_{2} z_{5}+\right. \\
& \left.+8 z_{6}+12 z_{1} z_{6}-4 z_{3} z_{6}+4 z_{6}^{2}-14 z_{2} z_{7}-5 z_{1} z_{2} z_{7}-9 z_{5} z_{7}-9 z_{7}^{2}+9 z_{1} z_{7}^{2}\right), \\
& a_{15}(z)=2\left(-12 z_{2}-6 z_{1} z_{2}+8 z_{5}+3 z_{1} z_{5}-5 z_{2} z_{6}+19 z_{7}+5 z_{1} z_{7}-5 z_{3} z_{7}-13 z_{6} z_{7}\right) \text {, } \\
& a_{16}(z)=4\left(9-3 z_{1}-3 z_{3}+2 z_{6}+z_{1} z_{6}-3 z_{2} z_{7}-9 z_{7}^{2}\right), \\
& a_{17}(z)=2\left(-7 z_{2}-19 z_{7}+z_{1} z_{7}\right) \text {, } \\
& a_{22}(z)=-40+24 z_{1}-36 z_{1}^{2}+7 z_{2}^{2}+24 z_{3}-4 z_{4}+44 z_{6}-16 z_{1} z_{6}-12 z_{2} z_{7}-36 z_{7}^{2}, \\
& a_{23}(z)=2\left(9 z_{2}-14 z_{1} z_{2}+4 z_{2} z_{3}+16 z_{5}-4 z_{1} z_{5}-5 z_{2} z_{6}+\right. \\
& \left.+4 z_{1} z_{7}-12 z_{1}^{2} z_{7}+7 z_{3} z_{7}-z_{6} z_{7}\right) \\
& a_{24}(z)=2\left(-7 z_{2}-4 z_{1} z_{2}-6 z_{1}^{2} z_{2}-z_{2} z_{3}+6 z_{2} z_{4}+20 z_{5}-6 z_{1} z_{5}-3 z_{3} z_{5}+\right. \\
& +14 z_{2} z_{6}-4 z_{1} z_{2} z_{6}-5 z_{5} z_{6}-10 z_{1} z_{7}+22 z_{1}^{2} z_{7}-6 z_{2}^{2} z_{7}- \\
& \left.-12 z_{3} z_{7}-5 z_{1} z_{3} z_{7}+12 z_{4} z_{7}-10 z_{6} z_{7}+13 z_{1} z_{6} z_{7}-9 z_{2} z_{7}^{2}\right), \\
& a_{25}(z)=40-24 z_{1}+12 z_{1}^{2}-14 z_{2}^{2}+12 z_{3}-12 z_{1} z_{3}+28 z_{4}+9 z_{2} z_{5}+16 z_{6}-12 z_{1} z_{6}- \\
& -8 z_{3} z_{6}-24 z_{6}^{2}+12 z_{2} z_{7}-10 z_{1} z_{2} z_{7}+14 z_{5} z_{7}-2 z_{7}^{2}-2 z_{1} z_{7}^{2},
\end{aligned}
$$




$$
\begin{aligned}
& a_{26}(z)=2\left(17 z_{2}-6 z_{1} z_{2}+8 z_{5}+3 z_{2} z_{6}-24 z_{1} z_{7}-5 z_{3} z_{7}-13 z_{6} z_{7}\right), \\
& a_{27}(z)=-48 z_{1}-12 z_{3}-28 z_{6}+3 z_{2} z_{7}, \\
& a_{33}(z)=4\left(-20+16 z_{1}-5 z_{1}^{2}-9 z_{1}^{3}+8 z_{3}+12 z_{1} z_{3}+3 z_{3}^{2}-7 z_{4}-\right. \\
& -z_{1} z_{4}-2 z_{2} z_{5}-9 z_{6}-3 z_{1} z_{6}-4 z_{1}^{2} z_{6}+2 z_{3} z_{6}-3 z_{6}^{2}+ \\
& \left.+6 z_{2} z_{7}-6 z_{1} z_{2} z_{7}+8 z_{5} z_{7}+z_{7}^{2}+7 z_{1} z_{7}^{2}\right), \\
& a_{34}(z)=2\left(-20-12 z_{1}-6 z_{1}^{2}+6 z_{1}^{3}+9 z_{2}^{2}-7 z_{1} z_{2}^{2}+20 z_{3}+6 z_{1} z_{3}-6 z_{1}^{2} z_{3}+\right. \\
& +6 z_{3}^{2}-26 z_{4}+6 z_{1} z_{4}+8 z_{3} z_{4}+2 z_{2} z_{5}-3 z_{1} z_{2} z_{5}-5 z_{5}^{2}-2 z_{6}-32 z_{1} z_{6}+ \\
& +20 z_{1}^{2} z_{6}-5 z_{2}^{2} z_{6}-24 z_{3} z_{6}-4 z_{1} z_{3} z_{6}+10 z_{4} z_{6}+4 z_{6}^{2}+4 z_{1} z_{6}^{2}- \\
& -3 z_{2} z_{7}-2 z_{1} z_{2} z_{7}-5 z_{1}^{2} z_{2} z_{7}-z_{2} z_{3} z_{7}+15 z_{5} z_{7}+ \\
& \left.+4 z_{2} z_{6} z_{7}+20 z_{7}^{2}-5 z_{1} z_{7}^{2}+5 z_{1}^{2} z_{7}^{2}+9 z_{3} z_{7}^{2}-9 z_{6} z_{7}^{2}\right), \\
& a_{35}(z)=2\left(-z_{2}+z_{1} z_{2}-6 z_{1}^{2} z_{2}-z_{2} z_{3}-22 z_{5}+15 z_{1} z_{5}+6 z_{3} z_{5}-\right. \\
& -15 z_{2} z_{6}-4 z_{1} z_{2} z_{6}-5 z_{5} z_{6}+18 z_{7}-5 z_{1} z_{7}+11 z_{1}^{2} z_{7}-6 z_{2}^{2} z_{7}-6 z_{3} z_{7}- \\
& \left.-5 z_{1} z_{3} z_{7}+12 z_{4} z_{7}+45 z_{6} z_{7}-8 z_{1} z_{6} z_{7}+12 z_{2} z_{7}^{2}-18 z_{7}^{3}\right), \\
& a_{36}(z)=2\left(20+6 z_{1}^{2}-7 z_{2}^{2}-18 z_{3}-6 z_{1} z_{3}+14 z_{4}+20 z_{6}+6 z_{1} z_{6}+\right. \\
& \left.+4 z_{3} z_{6}+6 z_{2} z_{7}-5 z_{1} z_{2} z_{7}-5 z_{5} z_{7}-z_{7}^{2}-13 z_{1} z_{7}^{2}\right), \\
& a_{37}(z)=2\left(-7 z_{2}-6 z_{1} z_{2}-5 z_{5}+19 z_{7}-13 z_{1} z_{7}+2 z_{3} z_{7}\right), \\
& a_{44}(z)=4\left(-10-16 z_{1}+8 z_{1}^{2}-6 z_{1}^{4}-4 z_{2}^{2}-16 z_{3}+24 z_{1}^{2} z_{3}-4 z_{2}^{2} z_{3}-12 z_{3}^{2}+\right. \\
& +8 z_{4}-16 z_{1} z_{4}-4 z_{1}^{2} z_{4}+8 z_{3} z_{4}+6 z_{4}^{2}+11 z_{2} z_{5}+2 z_{1} z_{2} z_{5}-z_{2} z_{3} z_{5}- \\
& -12 z_{5}^{2}-2 z_{1} z_{5}^{2}+4 z_{6}-4 z_{1} z_{6}-14 z_{1}^{2} z_{6}+4 z_{1}^{3} z_{6}+3 z_{2}^{2} z_{6}-2 z_{1} z_{2}^{2} z_{6}-4 z_{3} z_{6}- \\
& -8 z_{1} z_{3} z_{6}-2 z_{3}^{2} z_{6}+6 z_{4} z_{6}+4 z_{1} z_{4} z_{6}-3 z_{2} z_{5} z_{6}-2 z_{6}^{2}+4 z_{1} z_{6}^{2}+ \\
& +4 z_{3} z_{6}^{2}-2 z_{6}^{3}+z_{2} z_{7}+z_{1} z_{2} z_{7}+9 z_{1}^{2} z_{2} z_{7}-3 z_{2}^{3} z_{7}-8 z_{2} z_{3} z_{7}- \\
& -3 z_{1} z_{2} z_{3} z_{7}+9 z_{2} z_{4} z_{7}-21 z_{5} z_{7}+6 z_{1} z_{5} z_{7}+4 z_{1}^{2} z_{5} z_{7}-9 z_{3} z_{5} z_{7}- \\
& -6 z_{2} z_{6} z_{7}+7 z_{1} z_{2} z_{6} z_{7}+9 z_{5} z_{6} z_{7}-9 z_{7}^{2}+17 z_{1} z_{7}^{2}-3 z_{1}^{2} z_{7}^{2}- \\
& \left.-2 z_{1}^{3} z_{7}^{2}+9 z_{1} z_{3} z_{7}^{2}-9 z_{4} z_{7}^{2}+9 z_{6} z_{7}^{2}-9 z_{1} z_{6} z_{7}^{2}\right), \\
& a_{45}(z)=2\left(9 z_{2}-7 z_{1} z_{2}+13 z_{1}^{2} z_{2}-7 z_{2}^{3}-12 z_{2} z_{3}-7 z_{1} z_{2} z_{3}+21 z_{2} z_{4}-28 z_{5}-\right. \\
& -7 z_{1} z_{5}+7 z_{1}^{2} z_{5}-13 z_{3} z_{5}+9 z_{4} z_{5}-3 z_{2} z_{6}+7 z_{1} z_{2} z_{6}-3 z_{2} z_{3} z_{6}-19 z_{5} z_{6}- \\
& -4 z_{1} z_{5} z_{6}-5 z_{2} z_{6}^{2}+21 z_{1} z_{7}-24 z_{1}^{2} z_{7}+6 z_{1}^{3} z_{7}+7 z_{2}^{2} z_{7}-5 z_{1} z_{2}^{2} z_{7}+ \\
& +14 z_{3} z_{7}-z_{1} z_{3} z_{7}-5 z_{3}^{2} z_{7}-19 z_{4} z_{7}+10 z_{1} z_{4} z_{7}-z_{2} z_{5} z_{7}+ \\
& \left.+10 z_{1} z_{6} z_{7}+4 z_{1}^{2} z_{6} z_{7}+5 z_{6}^{2} z_{7}-2 z_{2} z_{7}^{2}+4 z_{1} z_{2} z_{7}^{2}+9 z_{5} z_{7}^{2}-9 z_{1} z_{7}^{3}\right), \\
& a_{46}(z)=2\left(20+12 z_{1}-18 z_{1}^{2}+12 z_{1}^{3}+7 z_{2}^{2}-6 z_{1} z_{2}^{2}+12 z_{3}-24 z_{1} z_{3}-6 z_{3}^{2}+2 z_{4}+\right. \\
& +12 z_{1} z_{4}-z_{2} z_{5}+2 z_{6}+8 z_{1} z_{6}+2 z_{1}^{2} z_{6}+8 z_{3} z_{6}+6 z_{4} z_{6}- \\
& -4 z_{6}^{2}-6 z_{2} z_{7}-z_{1} z_{2} z_{7}-4 z_{2} z_{3} z_{7}-10 z_{5} z_{7}-4 z_{1} z_{5} z_{7}-5 z_{2} z_{6} z_{7}- \\
& \left.-20 z_{7}^{2}+2 z_{1} z_{7}^{2}+4 z_{1}^{2} z_{7}^{2}-9 z_{3} z_{7}^{2}+9 z_{6} z_{7}^{2}\right), \\
& a_{47}(z)=2\left(-5 z_{2}-6 z_{1} z_{2}-5 z_{2} z_{3}-19 z_{5}-4 z_{1} z_{5}-5 z_{2} z_{6}-19 z_{7}+\right. \\
& \left.+11 z_{1} z_{7}+5 z_{1}^{2} z_{7}-10 z_{3} z_{7}+3 z_{4} z_{7}+9 z_{6} z_{7}\right),
\end{aligned}
$$




$$
\begin{aligned}
a_{55}(z)=- & 60+48 z_{1}-12 z_{1}^{2}-12 z_{1} z_{2}^{2}-24 z_{3}+24 z_{1} z_{3}-12 z_{3}^{2}-48 z_{4}+24 z_{1} z_{4}+ \\
& +12 z_{2} z_{5}+15 z_{5}^{2}-52 z_{6}+24 z_{1}^{2} z_{6}-48 z_{3} z_{6}-4 z_{4} z_{6}-48 z_{6}^{2}-16 z_{1} z_{6}^{2}+ \\
& +40 z_{2} z_{7}+8 z_{1} z_{2} z_{7}-8 z_{2} z_{3} z_{7}+20 z_{5} z_{7}+8 z_{1} z_{5} z_{7}-24 z_{2} z_{6} z_{7}-16 z_{7}^{2}+ \\
& +4 z_{1} z_{7}^{2}-12 z_{1}^{2} z_{7}^{2}+32 z_{3} z_{7}^{2}+28 z_{6} z_{7}^{2}, \\
a_{56}(z)=2\left(7 z_{2}-z_{1} z_{2}-5 z_{2} z_{3}+8 z_{5}+5 z_{1} z_{5}-7 z_{2} z_{6}+5 z_{5} z_{6}-28 z_{7}+19 z_{1} z_{7}-\right. & \\
& \left.-6 z_{1}^{2} z_{7}+11 z_{3} z_{7}-3 z_{4} z_{7}-17 z_{6} z_{7}-9 z_{1} z_{6} z_{7}-6 z_{2} z_{7}^{2}+9 z_{7}^{3}\right), \\
a_{57}(z)=- & 20+12 z_{1}-12 z_{3}-8 z_{4}-48 z_{6}-20 z_{1} z_{6}-12 z_{2} z_{7}+5 z_{5} z_{7}+20 z_{7}^{2}, \\
a_{66}(z)=4 & \left.-14+12 z_{1}-6 z_{1}^{2}+12 z_{3}-2 z_{4}+2 z_{6}+2 z_{6}^{2}-7 z_{2} z_{7}-z_{5} z_{7}-5 z_{7}^{2}-5 z_{1} z_{7}^{2}\right), \\
a_{67}(z)=2( & \left.-7 z_{2}-3 z_{5}-19 z_{7}-11 z_{1} z_{7}+2 z_{6} z_{7}\right), \\
a_{77}(z)=- & 60-24 z_{1}-4 z_{6}+3 z_{7}^{2} .
\end{aligned}
$$

Остается вычислить $b_{j}^{0}(z)+\kappa b_{j}^{1}(z)$. Для этого требуется некая новая информация: нам надо знать все симметрические мономы первого порядка для $E_{7}$ как функции переменных $z$. Чтобы получить их, мы используем разложения фундаментальных $E_{7}$-характеров в терминах мономиальных функций, вычисленных в работе [18]. В обозначениях работы [17] эти разложения имеют вид

$$
\begin{aligned}
z_{1}= & M_{\lambda_{1}}+7, \\
z_{2}= & M_{\lambda_{2}}+6 M_{\lambda_{7}}, \\
z_{3}= & M_{\lambda_{3}}+5 M_{\lambda_{6}}+22 M_{\lambda_{1}}+77, \\
z_{4}= & M_{\lambda_{4}}+4 M_{\lambda_{1}+\lambda_{6}}+15 M_{\lambda_{2}+\lambda_{7}}+15 M_{2 \lambda_{1}}+ \\
& \quad+45 M_{2 \lambda_{7}}+50 M_{\lambda_{3}}+145 M_{\lambda_{6}}+390 M_{\lambda_{1}}+980, \\
z_{5}= & M_{\lambda_{5}}+5 M_{\lambda_{1}+\lambda_{7}}+21 M_{\lambda_{2}}+71 M_{\lambda_{7}}, \\
z_{6}= & M_{\lambda_{6}}+6 M_{\lambda_{1}}+27, \\
z_{7}= & M_{\lambda_{7}} .
\end{aligned}
$$

Для обращения этих формул, имея целью вычисление фундаментальных мономиальных функций, мы должны действовать в порядке возрастания высоты доминантных весов, отвечающих характерам. Если мономиальная функция первого порядка известна, мы вычисляем соответствующие $b_{k}^{0}(z)$ и $b_{k}^{1}(z)$, следуя процедуре, описанной в работе [17]. Это позволяет на каждом шаге использовать уже известную часть оператора $\Delta^{\kappa}$ для вычисления мономиальных функций второго порядка заранее, т.е. до того как они понадобятся для получения следующей фундаментальной мономиальной функции. В рамках такого подхода легко найти

$$
\begin{aligned}
& M_{\lambda_{1}}=z_{1}-7 \\
& M_{\lambda_{2}}=z_{2}-6 z_{7} \\
& M_{\lambda_{3}}=z_{3}-5 z_{6}+8 z_{1}+2 \\
& M_{\lambda_{4}}=z_{4}-4 z_{1} z_{6}+9 z_{2} z_{7}+9 z_{1}^{2}+9 z_{7}^{2}-14 z_{3}-39 z_{6}-22 z_{1}-18 \\
& M_{\lambda_{5}}=z_{5}-5 z_{1} z_{7}+14 z_{2}+15 z_{7}
\end{aligned}
$$




$$
\begin{aligned}
& M_{\lambda_{6}}=z_{6}-6 z_{1}+15, \\
& M_{\lambda_{7}}=z_{7},
\end{aligned}
$$

а потому

$$
\begin{aligned}
& b_{1}^{0}(z)+\kappa b_{1}^{1}(z)=-28+4 z_{1}+\kappa\left(28+68 z_{1}\right), \\
& b_{2}^{0}(z)+\kappa b_{2}^{1}(z)= 7 z_{2}-24 z_{7}+\kappa\left(98 z_{2}+24 z_{7}\right), \\
& b_{3}^{0}(z)+\kappa b_{3}^{1}(z)=8-56 z_{1}+12 z_{3}-20 z_{6}+\kappa\left(-8+56 z_{1}+132 z_{3}+20 z_{6}\right), \\
& b_{4}^{0}(z)+\kappa b_{4}^{1}(z)=-+22+72 z_{1}-24 z_{1}^{2}+24 z_{3}+24 z_{4}-16 z_{6}-16 z_{1} z_{6}- \\
& \quad-24 z_{2} z_{7}+36 z_{7}^{2}+\kappa\left(72-72 z_{1}+24 z_{1}^{2}-24 z_{3}+\right. \\
&\left.\quad+192 z_{4}+16 z_{6}+16 z_{1} z_{6}+24 z_{2} z_{7}-36 z_{7}^{2}\right), \\
& b_{5}^{0}(z)+\kappa b_{5}^{1}(z)=-28 z_{2}+15 z_{5}-4 z_{7}-20 z_{1} z_{7}+\kappa\left(28 z_{2}+150 z_{5}+4 z_{7}+20 z_{1} z_{7}\right), \\
& b_{6}^{0}(z)+\kappa b_{6}^{1}(z)=-48-24 z_{1}+8 z_{6}+\kappa\left(48+24 z_{1}+104 z_{6}\right), \\
& b_{7}^{0}(z)+\kappa b_{7}^{1}(z)=3 z_{7}+54 \kappa z_{7} .
\end{aligned}
$$

Этим завершается вычисление $\Delta^{\kappa}$. В оставшейся части работы мы представим некоторые результаты, полученные с использованием этого оператора.

\section{3. НЕСКОЛЬКО ЯВНЫХ РЕЗУЛЬТАТОВ О НИЗКОЛЕЖАЩИХ СОБСТВЕННЫХ ФУНКЦИЯХ СИСТЕМ}

В этом разделе мы приведем некоторые результаты о многочленах первого и второго порядков и обобщенных квадратичных рядах Клебша-Гордана. Так как некоторые формулы слишком длинны, мы отсылаем заинтересованного читателя к работе [19], где приведены полные результаты.

3.1. Мономиальные симметрические функции второго порядка. Коль скоро мы знаем оператор $\Delta^{\kappa}$, можно вычислить его собственные функции с помощью итерационных алгоритмов, приведенных в работе [17]. В частности, можно получить мономиальные симметрические функции для $E_{7}$, просто полагая $\kappa=0$ в этих алгоритмах. Мы приведем здесь некоторые простейшие мономиальные функции второго порядка, полученные таким способом. Используем обозначение $M_{n_{1} \ldots n_{7}}=$ $M_{\sum_{j=1}^{7} n_{j} \lambda_{j}}$. Тогда

$$
\begin{aligned}
M_{2000000}=z_{1}^{2}-2 z_{3}-2 z_{1}-7 \\
M_{1100000}=z_{1} z_{2}-5 z_{5}+3 z_{1} z_{7}-23 z_{7} \\
M_{0200000}=z_{2}^{2}-2 z_{4}-2 z_{2} z_{7}-2 z_{1}^{2}-6 z_{7}^{2}+4 z_{3}+14 z_{6}+4 z_{1}+12, \\
M_{1010000}=z_{1} z_{3}-3 z_{4}-z_{1} z_{6}+6 z_{2} z_{7}-3 z_{1}^{2}-9 z_{7}^{2}-9 z_{3}+4 z_{6}+20 z_{1}+32, \\
M_{0110000}=z_{2} z_{3}-4 z_{1} z_{5}+5 z_{2} z_{6}+4 z_{1}^{2} z_{7}-4 z_{3} z_{7}-17 z_{1} z_{2}- \\
\quad \quad-16 z_{6} z_{7}+41 z_{5}+13 z_{1} z_{7}+12 z_{2}+7 z_{7} \\
M_{0000020}=z_{6}^{2}-2 z_{5} z_{7}+2 z_{4}-6 z_{1}^{2}-2 z_{7}^{2}+12 z_{3}+4 z_{6}+12 z_{1}+17, \\
M_{1000001}=z_{1} z_{7}-7 z_{2}+8 z_{7},
\end{aligned}
$$




$$
\begin{aligned}
& M_{0100001}=z_{2} z_{7}-6 z_{3}-6 z_{7}^{2}+14 z_{6}+16 z_{1}-28 \\
& M_{0010001}=z_{3} z_{7}-5 z_{6} z_{7}-6 z_{1} z_{2}+20 z_{5}+24 z_{1} z_{7}-49 z_{2}-12 z_{7}, \\
& M_{0000101}=z_{5} z_{7}-4 z_{4}-5 z_{1} z_{7}^{2}+8 z_{1} z_{6}+11 z_{2} z_{7}+12 z_{1}^{2}-3 z_{7}^{2}-42 z_{3}-18 z_{6}-4, \\
& M_{0000011}=z_{6} z_{7}-3 z_{5}-z_{1} z_{7}+7 z_{2}-11 z_{7} \\
& M_{0000002}=z_{7}^{2}-2 z_{6}-2 .
\end{aligned}
$$

\section{2. Разложение характеров второго порядка по мономиальным функ-} циям. Как можно заключить уже из названия, ортогональная система мономиальных симметрических функций является простейшей среди различных систем симметрических многочленов, связанных с алгеброй Ли $E_{7}$ : каждая мономиальная симметричная функция является не чем иным, как суммой всех мономов, связанных с одной орбитой группы Вейля на решетке весов. Теперь легко разложить другие многочлены, связанные с системой корней $E_{7}$, по базису мономиальных симметрических функций. На самом деле, метод совпадает с тем, который мы описали в работе [17] для вычисления рядов Клебша-Гордана. В частности, коэффициенты в разложении характеров по мономиальным симметрическим функциям интересны тем, что они дают кратности весов в соответствующих неприводимых представлениях. В качестве примера приведем такое разложение для нескольких характеров второго порядка:

$$
\begin{aligned}
& \chi_{2000000}=M_{2000000}+M_{0010000}+4 M_{0000010}+17 M_{1000000}+63 M_{0000000}, \\
& \chi_{1100000}=M_{1100000}+4 M_{0000100}+16 M_{1000001}+56 M_{0100000}+171 M_{0000001} \text {, } \\
& \chi_{0200000}=M_{0200000}+M_{0001000}+3 M_{1000010}+11 M_{0100001}+10 M_{2000000}+ \\
& +36 M_{0000002}+34 M_{0010000}+96 M_{0000010}+ \\
& +248 M_{1000000}+603 M_{0000000}, \\
& \chi_{1010000}=M_{1010000}+2 M_{0001000}+8 M_{1000010}+24 M_{0100001}+32 M_{2000000}+ \\
& +64 M_{0000002}+78 M_{0010000}+208 M_{0000010}+ \\
& +544 M_{1000000}+1344 M_{0000000}, \\
& \chi_{0110000}=M_{0110000}+3 M_{1000100}+10 M_{0100010}+10 M_{2000001}+30 M_{0010001}+ \\
& +90 M_{1100000}+80 M_{0000011}+231 M_{0000100}+570 M_{1000001}+ \\
& +1344 M_{0100000}+3024 M_{0000001}, \\
& \chi_{0000101}=M_{0000101}+3 M_{0001000}+5 M_{1000002}+13 M_{1000010}+45 M_{0100001}+ \\
& +39 M_{2000000}+135 M_{0000002}+129 M_{0010000}+ \\
& +351 M_{0000010}+879 M_{1000000}+2079 M_{0000000}, \\
& \chi_{0000011}=M_{0000011}+2 M_{0000100}+10 M_{1000001}+35 M_{0100000}+111 M_{0000001} \text {, } \\
& \chi_{0000002}=M_{0000002}+M_{0000010}+5 M_{1000000}+21 M_{0000000} \text {. }
\end{aligned}
$$

3.3. Некоторые многочлены первого и второго порядков. Итерационные методы, изложенные в [17], позволяют решить уравнение (4) на собственные значения при произвольных $\kappa$. Собственные функции являются многочленами. Здесь мы 
приведем некоторые из этих многочленов первого и второго порядков. Как было замечено выше, после умножения на $\Psi_{0}^{\kappa}$ эти многочлены дают волновые функции исходной квантово-механической задачи

$$
H \Psi_{\mathbf{m}}^{\kappa}=E_{\mathbf{m}}(\kappa) \Psi_{\mathbf{m}}^{\kappa}
$$

с гамильтонианом (1) и уровнями энергии, заданными как $E_{\mathbf{m}}(\kappa)=2(\mu+\kappa \rho, \mu+$ $\kappa \rho)$ (см. подробности в [17]). Для выражения волновых функций в терминах переменных $q$ (координат частиц) следует помнить, что $z_{k}=\sum_{\lambda} e^{2 i(\lambda, q)}$, где суммирование идет по весам $\lambda$ неприводимых представлений, связанных с характером $z_{k}$. Если волновые функции известны, можно обратиться к таким стандартным квантово-динамическим задачам, как вычисление ожидаемого значения координат и импульсов или построенных из них наблюдаемых, амплитуд перехода между уровнями энергии при малых возмущениях гамильтониана и др.

Многочлены первого и второго порядков имеют вид

$$
\begin{aligned}
& P_{1000000}^{\kappa}(z)=z_{1}+\frac{7(-1+\kappa)}{1+17 \kappa}, \\
& P_{0100000}^{\kappa}(z)=z_{2}+\frac{6(-1+\kappa) z_{7}}{1+11 \kappa}, \\
& P_{0010000}^{\kappa}(z)=z_{3}+\frac{5(-1+\kappa) z_{6}}{1+7 \kappa}+\frac{8(-1+\kappa)(-1+8 \kappa) z_{1}}{(1+7 \kappa)(1+8 \kappa)}+ \\
& +\frac{2(-1+\kappa)\left(-1-159 \kappa+136 \kappa^{2}\right)}{(1+7 \kappa)(1+8 \kappa)(1+11 \kappa)}, \\
& P_{0000100}^{\kappa}(z)=z_{5}+\frac{5(-1+\kappa) z_{1} z_{7}}{1+7 \kappa}+\frac{7(-1+\kappa)(-4+7 \kappa) z_{2}}{(1+7 \kappa)(2+13 \kappa)}+ \\
& +\frac{5(-1+\kappa)\left(-6-137 \kappa+56 \kappa^{2}\right) z_{7}}{(1+7 \kappa)(1+8 \kappa)(2+13 \kappa)}, \\
& P_{0000010}^{\kappa}(z)=z_{6}+\frac{6(-1+\kappa) z_{1}}{1+9 \kappa}+\frac{15(-1+\kappa)(-1+5 \kappa)}{(1+9 \kappa)(1+13 \kappa)}, \\
& P_{0000001}^{\kappa}(z)=z_{7}, \\
& P_{2000000}^{\kappa}(z)=z_{1}^{2}+\frac{-2 z_{3}}{1+\kappa}+\frac{-10 \kappa z_{6}}{(1+\kappa)(1+4 \kappa)}+\frac{2\left(-3-6 \kappa-119 \kappa^{2}+28 \kappa^{3}\right) z_{1}}{(1+\kappa)(1+4 \kappa)(3+17 \kappa)}+ \\
& +\frac{-42-459 \kappa-290 \kappa^{2}-3205 \kappa^{3}+196 \kappa^{4}}{(1+\kappa)(1+4 \kappa)(2+17 \kappa)(3+17 \kappa)}, \\
& P_{1100000}^{\kappa}(z)=z_{1} z_{2}+\frac{-5 z_{5}}{1+4 \kappa}+\frac{\left(6-95 \kappa+24 \kappa^{2}\right) z_{1} z_{7}}{(1+4 \kappa)(2+11 \kappa)}+\frac{28(-1+\kappa) \kappa(-26+11 \kappa) z_{2}}{(1+4 \kappa)(2+11 \kappa)(3+17 \kappa)}+ \\
& +\frac{(-1+\kappa)\left(138+365 \kappa-9979 \kappa^{2}+1176 \kappa^{3}\right) z_{7}}{(1+4 \kappa)(1+7 \kappa)(2+11 \kappa)(3+17 \kappa)}, \\
& P_{1000001}^{\kappa}(z)=z_{1} z_{7}+\frac{-7 z_{2}}{1+6 \kappa}+\frac{\left(16-191 \kappa+42 \kappa^{2}\right) z_{7}}{(1+6 \kappa)(2+17 \kappa)}, \\
& P_{0000011}^{\kappa}(z)=z_{6} z_{7}+\frac{-3 z_{5}}{1+2 \kappa}+\frac{\left(-2-43 \kappa+12 \kappa^{2}\right) z_{1} z_{7}}{(1+2 \kappa)(2+9 \kappa)}+\frac{-7(-1+\kappa)(2+19 \kappa) z_{2}}{(1+2 \kappa)(1+5 \kappa)(2+9 \kappa)}+
\end{aligned}
$$




$$
+\frac{2(-1+\kappa)\left(22-2 \kappa-1052 \kappa^{2}+375 \kappa^{3}\right) z_{7}}{(1+2 \kappa)(1+5 \kappa)(2+9 \kappa)(2+13 \kappa)},
$$

$$
P_{0000002}^{\kappa}(z)=z_{7}^{2}+\frac{-2 z_{6}}{1+\kappa}+\frac{-12 \kappa z_{1}}{(1+\kappa)(1+5 \kappa)}+\frac{-2\left(1+59 \kappa^{2}\right)}{(1+\kappa)(1+5 \kappa)(1+9 \kappa)} \text {. }
$$

3.4. Обобщенный квадратичный ряд Клебша-Гордана. Для произвольного $\kappa$ произведение многочленов можно разложить в линейную комбинацию многочленов при том же $\kappa$. В это разложение входят ровно те члены, которые входят в произведение характеров, т.е. в соответствующий ряд Клебша-Гордана, а коэффициенты являются рациональными функциями $\kappa$. Метод вычисления этих коэффициентов был изложен в работе [17]. Мы приведем здесь некоторые из квадратичных рядов Клебша-Гордана при общих значениях $\kappa$.

Разумеется, результаты этого раздела не зависят от переменных, используемых для записи многочленов. Тем не менее $W$-инвариантность задачи является важным обстоятельством, а потому наш выбор переменных $z$ вполне естественен. Заметим, что в этих переменных гамильтониан есть дифференциальный оператор, коэффициенты которого являются многочленами, тогда как другой выбор переменных приводит к рациональным функциям, в последнем случае анализ значительно проще. Однако $W$-инвариантность является ключевым фактором. Можно было бы использовать и другие наборы переменных, также обладающие этой инвариантностью, например, мономиальные симметрические функции.

Интерес к приведенным здесь результатам является двояким. Во-первых, с чисто математической точки зрения они задают деформацию ряда Клебша-Гордана для $E_{7}$ в зависимости от параметра $\kappa$. Во-вторых, они могли бы быть полезны и при исследовании ряда физических задач. Например, в нашей работе [13] представлен пертурбативный подход к эллиптической системе Калоджеро-Сазерленда, связанной с алгеброй $A$ наинизшего ранга, в которой использование рекурсионных соотношений такого типа играет определяющую роль.

Итак, мы имеем

$$
\begin{aligned}
P_{1000000}^{\kappa} \times P_{1000000}^{\kappa}=P_{2000000}^{\kappa}+\frac{2}{1+\kappa} P_{0010000}^{\kappa}+\frac{10(1+3 \kappa)}{(1+4 \kappa)(1+7 \kappa)} P_{0000010}^{\kappa}+ & \\
& +\frac{24\left(4+103 \kappa+547 \kappa^{2}+696 \kappa^{3}\right)}{(1+8 \kappa)(1+9 \kappa)(1+17 \kappa)(3+17 \kappa)} P_{1000000}^{\kappa}+ \\
& +\frac{252(1+3 \kappa)(1+5 \kappa)(1+8 \kappa)(1+18 \kappa)}{(1+11 \kappa)(1+13 \kappa)(1+17 \kappa)^{2}(2+17 \kappa)}, \\
P_{1000000}^{\kappa} \times P_{0100000}^{\kappa}=P_{1100000}^{\kappa}+\frac{5}{1+4 \kappa} P_{0000100}^{\kappa}+ & \\
+ & \frac{32(1+2 \kappa)(1+12 \kappa)}{(1+7 \kappa)(1+11 \kappa)(2+11 \kappa)} P_{1000001}^{\kappa}+ \\
& +\frac{42(1+3 \kappa)(1+14 \kappa)\left(5+101 \kappa+74 \kappa^{2}\right)}{(1+6 \kappa)(1+11 \kappa)(2+13 \kappa)(1+17 \kappa)(3+17 \kappa)} P_{0100000}^{\kappa}+ \\
& +\frac{144(1+2 \kappa)(1+3 \kappa)(1+5 \kappa)(1+18 \kappa)}{(1+7 \kappa)(1+8 \kappa)(1+11 \kappa)^{2}(2+17 \kappa)} P_{0000001}^{\kappa},
\end{aligned}
$$




$$
\begin{aligned}
P_{1000000}^{\kappa} \times P_{0000001}^{\kappa}= & P_{1000001}^{\kappa}+\frac{7}{1+6 \kappa} P_{0100000}^{\kappa}+ \\
& +\frac{54(1+3 \kappa)(1+18 \kappa)}{(1+11 \kappa)(1+17 \kappa)(2+17 \kappa)} P_{0000001}^{\kappa}, \\
P_{0100000}^{\kappa} \times P_{0000001}^{\kappa}=P_{0100001}^{\kappa}+\frac{6}{1+5 \kappa} P_{0010000}^{\kappa}+ & \\
+ & \frac{16(1+2 \kappa)(1+13 \kappa)}{(1+6 \kappa)(1+7 \kappa)(1+11 \kappa)} P_{0000010}^{\kappa}+ \\
& +\frac{32(1+2 \kappa)(1+4 \kappa)(1+17 \kappa)}{(1+7 \kappa)(1+8 \kappa)(1+9 \kappa)(1+11 \kappa)} P_{1000000}^{\kappa}, \\
P_{0000001}^{\kappa} \times P_{0000001}^{\kappa}=P_{0000002}^{\kappa}+\frac{2}{1+\kappa} P_{0000010}^{\kappa}+\frac{12(1+4 \kappa)}{(1+5 \kappa)(1+9 \kappa)} P_{1000000}^{\kappa}+ & +\frac{56(1+4 \kappa)(1+8 \kappa)}{(1+9 \kappa)(1+13 \kappa)(1+17 \kappa)} .
\end{aligned}
$$

\section{4. ЗАКЛЮЧЕНИЕ И ЗАМЕЧАНИЯ}

Мы получили некоторые явные результаты для системы Калоджеро-Сазерленда, которая связана с исключительной алгеброй Ли $E_{7}$. Ключевым моментом наших рассуждений было вычисление гамильтониана в терминах характеров фундаментальных представлений. Для этого вычисления мы использовали нашу предыдущую работу [17] и разложение переменных $z$ в терминах мономиальных симметрических функций. Когда гамильтониан известен, открывается широкое поле приложений. Мы привели два: некоторые многочлены первого и второго порядка и некоторые обобщенные квадратичные ряды Клебша-Гордана.

Мы полагаем, что представленное в данной статье направление исследований подтверждает полезность нашего подхода для получения явных результатов о системах Калоджеро-Сазерленда в духе предыдущих статей [7], [16], [17].

Благодарности. Работа была частично поддержана со стороны испанского Мinisterio de Educación y Ciencia (гранты MTM2006-10531 (Х. Фернандес Нуньес) и FIS2006-09417 (В. Гарсиа Фуертес и А. М. Переломов)). Статья была закончена во время визита А. М. Переломова в Институт гравитационной физики Макса Планка; автор благодарит научное руководство этого института за поддержку.

\section{Список литературы}

[1] F. Calogero, J. Math. Phys., 12 (1971), 419-436.

[2] B. Sutherland, Phys. Rev. A, 4 (1972), 2019-2021.

[3] M. A. Olshanetsky, A. M. Perelomov, Lett. Math. Phys., 2 (1977), 7-13.

[4] М.А. Ольшанецкий, А.М. Переломов, Функи. анализ и его прилож., 12:2 (1978), $57-65$.

[5] M. A. Olshanetsky, A. M. Perelomov, Phys. Rep., 94 (1983), 313-404.

[6] S. Helgason, Differential Geometry, Lie Groups and Symmetric Spaces, Pure Appl. Math., 80, Academic Press, New York-San Francisco-London, 1978; Groups and Geometric Analysis. Integral Geometry, Invariant Differential Operators, and Spherical Functions, Pure Appl. Math., 113, Academic Press, Orlando, 1984. 
[7] A. M. Perelomov, J. Phys. A, 31 (1998), L31-L37.

[8] A. M. Perelomov, E. Ragoucy, Ph. Zaugg, J. Phys. A, 31 (1998), L559-L565.

[9] A. M. Perelomov, J. Phys. A, 32 (1999), 8563-8576.

[10] A. M. Perelomov, "Quantum integrable systems and special functions", Lie Theory and Its Applications in Physics. III (Clausthal, Germany, 1999), World Sci. Publ., Singapore, 2000, 139-154; arXiv: math-ph/0111021.

[11] W. García Fuertes, M. Lorente, A. M. Perelomov, J. Phys. A, 34 (2001), 10963-10973.

[12] В. Гарсия Фуертес, А.М. Переломов, ТМФ, 131:2 (2002), 194-196; arXiv: math-ph/0201026.

[13] J. Fernández Núñez, W. García Fuertes, A. M. Perelomov, Phys. Lett. A, 307 (2003), 233-238.

[14] J. Fernández Núñez, W. García Fuertes, A. M. Perelomov, J. Math. Phys., 44 (2003), 4957-4974.

[15] J. Fernández Núñez, W. García Fuertes, A. M. Perelomov, J. Nonlin. Math. Phys., 12:1-suppl. (2005), 280-301.

[16] J. Fernández Núñez, W. García Fuertes, A. M. Perelomov, J. Math. Phys., 46 (2005), 073508 .

[17] J. Fernández Núñez, W. García Fuertes, A. M. Perelomov, J. Math. Phys., 46 (2005), 103505.

[18] I. Loris, R. Sasaki, J. Phys. A, 37 (2004), 211-237.

[19] J. Fernández Núñez, W. García Fuertes, A. M. Perelomov, Explicit computations of low lying eigenfunctions for the quantum trigonometric Calogero-Sutherland model related to the exceptional algebra E7, arXiv: math-ph/0604062.

Поступила в редакцию 18.03.2007, после доработки 13.04.2007 